\title{
Motifs et manifestations de l'amour de l'homme pour Dieu et l'amour du prochain selon Saint Basile le Grand
}

\author{
Motives and examples of human love for God \\ and the neighbour according to Saint Basil the Great
}

\begin{abstract}
This article addresses the issue of the motivation of the fundamental order of love for God and the neighbour which Saint Basil the Great used in his doctrine.

Based on an analysis of the texts of Saint Basil, this article demonstrates the intimate relationship that should exist between the love for God and the love for man. The ideal of this relationship is represented, according to the analysis, by Jesus Christ, the only Saviour of the world.
\end{abstract}

\section{Keywords}

Motive, the Saviour of the world, prayer, contemplation, imitation, the Creator, the Holy Spirit, The Basiliad.

\section{Introduction}

Lamour de l'homme pour son Créateur est une réponse à l'amour précédemment manifesté par Dieu. Quelle sera cette réponse dépendra de l' homme lui-même. Aimer Dieu se reflète dans les attitudes, les croyances, les comportements et les actions de l'homme. 
Le $I^{\mathrm{e}}$ siècle de l'ère chrétienne est l'un des plus importants dans le développement de la pensée théologique. Ce temps commence par un événement très important : le premier concile œcuménique de Nicée en 325.

Le but de cet article est de dégager et expliciter l'argumentation que saint Basile a utilisée dans le cadre de l'ordre fondamental de l'amour pour Dieu et pour autrui. Les conclusions résultant de l'analyse des textes de Basile le Grand ont été données à la fin de cet article.

\section{Motifs et manifestations de l'amour de l'homme pour Dieu}

Aimé par Dieu du plus grand amour, l'homme est capable, réciproquement, d'aimer Dieu en retour. L'amour et le désir de Dieu sont dans la nature de l'homme. ${ }^{1}$ Par conséquent, tout le monde doit aimer Dieu. Et cet amour pour Dieu implique pour l'homme de vouloir connaitre Dieu, le contempler, le voir... en un mot, le désirer avec ardeur. ${ }^{2}$

Basile estime que l'une des manifestations de l'amour de l'homme pour Dieu est de garder ses commandements. Il cite à cet égard l'Évangile selon saint Jean: "Celui qui a mes commandements et qui les garde, c'est celui-là qui m’aime ». ${ }^{3}$ Comme preuve de son amour pour lui, Dieu demande à l'homme de garder ses commandements. ${ }^{4}$

La nature de l'homme est d'aimer ce qui est bon et beau ; il n'a pas besoin d'apprendre à aimer son Créateur. Ceci est aussi naturel que l'amour pour ceux qui nous ont donné la vie, élevés ou donné à manger. «C'est par nature que nous voulons le bien, bien que généralement tout le monde l'imagine autrement $»^{5}$ Le fait que chaque homme puisse concevoir de manière différente ce que cette valeur représente, peut être source de confusion.

Dieu satisfait les critères du bien et de la beauté. Il est tout à la fois la plénitude du bien, du beau et du vrai. Il constitue le critère parfait de ces valeurs. L'homme

${ }^{1}$ Cf. J. Pryszmont, Patrystyczna myśl moralna, „Studia Theologica Varsaviensia” 2 (1981), p. 133.

${ }^{2}$ Cf. T. Spidlik, I. Gargano, Duchowość ojców greckich i wschodnich, Kraków 1997, p. 53.

${ }^{3} \mathrm{~J} 14,21$.

${ }^{4}$ Cf. Reguly Krótsze 213.

${ }^{5}$ Reguty Dtuższe 2,1. 
qui désire de même ces valeurs aspire à Dieu, et il l'aime. ${ }^{6}$ Et dans la recherche de la vérité, le Saint-Esprit est une aide précieuse pour l'homme. Il éclaire l'homme, il l'instruit et le conduit jusqu'à la vérité tout entière. ${ }^{7}$ Il conduit également l'homme à connaître le Père par l'intermédiaire du Fils. ${ }^{8}$

Dieu, dans la vie humaine, doit avoir en toute chose la primauté. Il est pour l'homme la source et le but de la vie. L'homme et sa nature sont parfaits seulement quand ils restent en communion avec Dieu et quand ils sont remplis de l'Esprit Saint. ${ }^{9}$ En éclairant l' homme, le Saint-Esprit le rend conforme à Dieu, son Créateur. C'est un processus de déification de l'homme. Ce processus, cependant, exige un effort humain, l'ascétisme et la contemplation de Dieu. ${ }^{10}$

L'amour de l'homme pour Dieu est influencé par la prise de conscience des œuvres de Dieu pour son salut, révélées dans la personne du Christ. L'évêque de Césarée cite un passage de la Lettre de saint Paul aux Philippiens, et il l'interprète. «Le Christ Jésus, ayant la condition de Dieu, ne retint pas jalousement le rang qui l'égalait à Dieu. Mais il s'est anéanti, prenant la condition de serviteur, devenant semblable aux hommes. Reconnu homme à son aspect, il s'est abaissé, devenant obéissant jusqu'à la mort, et la mort de la croix $»{ }^{11}$

Voilà le commentaire de Basile sur ce passage: « Alors, quand l'âme croit en ces paroles et à ce qu'elles expriment, alors elle apprend à connaître la grandeur de Sa gloire, s'émerveillant de l'immense humilité et de l'obéissance manifestées par le Christ qui, étant si grand, est devenu pour nos vies obéissant au Père jusqu'à la mort. C'est alors que naît dans l'âme humaine l'amour pour Dieu le Père, « qui n’a point épargné son propre Fils, mais qui l’a livré pour nous tous », ${ }^{12}$ en regard de l’amour manifesté par Dieu dans le Christ ». ${ }^{13}$

L'amour de l'homme pour Dieu se pose quand une personne contemple l'humilité de Dieu dans l' humilité et l'obéissance du Christ dans l'accomplissement de la volonté de son Père. Il se pose quand une personne doit faire face

${ }^{6}$ Reguly Dłuższe 2,1.

7 Cf. W. Hryniewicz, Człowiek w mocy Ducha Świętego. Zarys pneumatologii Ojców Wschodnich, „Znak” 29 (1977), p. 786.

8 W. Hryniewicz, Człowiek w mocy Ducha Świętego, p. 792.

9 Cf. J. Meyendorff, Teologia bizantyjska, Warszawa 1984, p. 216.

${ }^{10}$ Cf. W. Hryniewicz, Człowiek w mocy Ducha Świętego, p. 785, 786.

11 Flp 2,6-8.

$12 \mathrm{Rz} 8,32$.

${ }^{13}$ Reguly Krótsze 172. 
à l'amour de Dieu. Nous pouvons dire que l'amour de Dieu fait naître l'amour de l'homme d'une manière naturelle, et qu'une telle réaction de l'homme est une réponse correcte, et logiquement la seule possible. L'amour pour Dieu est né de l'admiration pour sa bonté et sa gloire.

Il est important de noter cette distinction qu'opère Basile sur deux types possibles de notre relation à Dieu : l'homme peut soit aimer Dieu, soit le craindre. Il motive ainsi cette distinction: «Celui qui fait que le soleil se lève, qui donne la rosée, fait également tomber la pluie de feu. L’un est un signe de sa bonté, les autres de son austérité. Soit nous aimons à cause de la première, soit nous avons peur à cause de ce dernier $»{ }^{14}$

Pour l'auteur de Reguly, seul l'amour qui naît de la gratitude pour les bienfaits de Dieu peut être qualifié de total et précieux. Dieu, en tant que beauté parfaite, se révèle être le plus grand désir de lâme. Sur ce point, la pensée de Basile s'exprime remarquablement dans la règle Reguła Dłuższa 2:1: "Qu'est-ce qui est plus admirable que la beauté de Dieu? Quelle pensée plus reconnaissante que celle de la splendeur de Dieu ? Quel désir aussi fort et ininterrompu, que de voir Dieu accorder à l'âme purifiée de tout mal et qui crie au milieu de l'extase: " Je suis blessé d'amour " ? $^{15}$ Totalement indescriptibles et indicibles sont les lueurs de la beauté divine. La langue ne les exprimera pas, ni l'oreille ne les comprendra ». Si vous les appelez « lumière de l'astre du jour » ou « luminosité de la lune ou du soleil », tout cela n'est rien par rapport à la gloire de Dieu, et la comparaison avec la vraie lumière est plus éloignée d’elle que la nuit profonde et sombre ne l'est de midi. ${ }^{16}$

Dans ce passage s'exprime déjà l'amour de Basile pour Dieu. C'est un ravissement devant la splendeur de Dieu, devant sa bonté et sa beauté, infinies et ineffables.

L'amour pour Dieu est une obligation directe de l'homme. Cette obligation découle de l'abondance des dons reçus. Basile donc explique son point de vue: « Si, par un réflexe naturel, nous sommes saisis par un sentiment de bonté et d'amour pour nos bienfaiteurs, et si nous faisons tous les efforts possibles pour rendre la pareille en regard du bien reçu, alors est-il seulement un mot par lequel nous pouvons exprimer notre gratitude pour les dons de Dieu ? Ils sont si abondants que personne ne peut les compter, si grands et de telle nature

\footnotetext{
${ }^{14}$ Reguly Dłuższe, Wstęp 4.

15 Pnp 2,5.

${ }^{16}$ Reguly Dłuzsze 2,1.
} 
que même un seul suffit pour nous obliger à une gratitude immense envers le Donateur $»{ }^{17}$

Le motif premier et principal de l'amour pour Dieu est la création elle-même. La chose la plus précieuse que l'homme possède est sans aucun doute la vie. Dieu est celui qui donne la vie, qui crée les êtres. L'homme a donc envers Dieu une dette, en paiement de sa propre création. La nature de l'homme est de rendre grâces au bienfaiteur pour le don reçu. ${ }^{18}$ Dans ce cas, le don est unique ; la réponse humaine à un don aussi précieux ne peut donc être que l'amour pour le Créateur.

«Ne soyons pas des étrangers et sans cœur à Celui qui nous a créés! Si vous ne l'avez pas connu comme il est, sur la base de sa bonté, alors, ne fût-ce que pour la seule raison que c'est Lui qui nous a créés, nous devons l'aimer sans mesure, et être constamment prêts à faire mémoire de Lui ». ${ }^{19}$

L'homme, par le seul fait de sa création, est obligé d'aimer son Créateur, et à faire toujours mémoire de lui. Basile dans Reguły n’approfondit pas plus loin le thème de l'amour. Cela prouve cependant - ou plutôt seulement - que pour lui, l'argument est si évident qu'il ne nécessite nulle autre explication. Basile qualifie tout simplement l'amour de Dieu de « dette nécessaire ». ${ }^{20}$

Exprimant son amour, l'homme doit servir Dieu. Cela doit être un service avec une "bonne disposition " et la volonté de plaire à Dieu. Ce que cela signifie exactement est explicité dans la Reguła Krótsza, au \$157 : «Une bonne disposition est un désir ardent, insatiable, fort et constant de plaire à Dieu ». Il est obtenu par la persévérance à considérer la grandeur de la gloire de Dieu, et par la pensée reconnaissante et la mémoire permanente des bénédictions reçues de Dieu. ${ }^{21}$

Le culte envers Dieu doit être rendu à lui-même, et pour sa gloire. Ceci doit être le principal motif du culte rendu à Dieu. Par la mémoire de toutes les bénédictions reçues de Dieu, l'homme lui rend grâces pour sa générosité et son amour. Des avantages au regard desquels nous devrions montrer une attitude de gratitude. $^{22}$

\footnotetext{
17 Reguły Dłuższe 2,2.

${ }^{18}$ Cf. Reguly Dluższe 2,1.

19 Reguty Dtuższe 2,2.

${ }^{20}$ Reguły Dłuższe 2,2.

21 Reguly Krótsze 157.

22 Cf. Reguty Krótsze 212.
} 
«Si nous sommes conscients des avantages, et si nous en sommes reconnaissants, n'allons-nous pas aimer Dieu, qui nous fait tant de grandes et nombreuses faveurs? Une telle attitude naît dans une âme saine, pour ainsi dire, d'une manière naturelle et sans apprentissage $»^{23}$

L'amour pour Dieu est naturel; il ne s'acquiert pas au fil du temps. Il est le propre d'une « âme saine. » Un homme qui ne serait pas en mesure d'éprouver ce sentiment se trouverait irait à l'encontre de sa propre nature.

Basile croit que si l'homme aime Dieu en vérité, il fera tout cela et à tout moment pour sa gloire. ${ }^{24}$ Il cite à ce titre la Première Lettre de saint Paul aux Corinthiens: "Soit donc que vous mangiez, soit que vous buviez, soit que vous fassiez quelque autre chose, faites tout pour la gloire de Dieu $» .^{25}$ Commentant ces paroles, l'évêque de Césarée écrit : "De cette façon, un homme pieux, ne cherchant ni présent ni la gloire future, mais seulement l'amour de Dieu et du prochain aurait l'audace de dire: « J'ai l'assurance que ni la mort ni la vie, ni les anges ni les dominations, ni les choses présentes ni les choses à venir, ni les puissances, ni la hauteur, ni la profondeur, ni aucune autre créature ne pourra nous séparer de l'amour de Dieu manifesté en Jésus Christ notre Seigneur ${ }^{26}{ }^{26}$

L'amour de Dieu doit être pour l'homme la chose la plus importante. Rien ne peut venir obscurcir cet amour. Son amour pour le prochain, l'homme peut le montrer à bien des égards. La forme la plus visible et précieuse sont les actes, car ils sont l'expression maximale de l'homme. ${ }^{27}$ Ils agissent également comme facteurs d'édification des autres. ${ }^{28}$ Selon le proverbe, même si les mots apprennent, les exemples attirent (latin : verba docent, exempla trahunt). Tout d'abord les actions prouvent que l'on aime quelqu'un. Lamour sans œuvres n'est pas un amour complet.

La prière doit être présente dans la vie humaine, car elle est l'expression de l'amour pour le Créateur, et la source de la divinisation de l'homme, en tant qu'il se conforme par elle au Créateur. ${ }^{29}$ Son essence est la « montée vers

\footnotetext{
${ }^{23}$ Reguly Krótsze 212.

${ }^{24}$ Reguly Krótsze 138.

251 Kor $10,31$.

${ }^{26}$ Reguly Krótsze 299.

27 Cf. Homilia in divites 6, „Patrologia Graeca” 31, 296 C.

28 List 150,4.

${ }^{29}$ Cf. W. Hryniewicz, Człowiek w mocy Ducha Świętego, p. 785.
} 
Dieu de l'âme et du cœur. Il faut prier sans cesse ${ }^{30}$ Saint Basile a développé le concept de la prière en tant que mémoire continue à Dieu. Cette mémoire est étroitement liée à l'amour de Dieu, elle purifie (personnification interne) et stimule le désir de " plaire à Dieu ». La prière incessante est, en quelque sorte, une « contemplation dans l'action ${ }^{31}$ par volonté constante et un "sincère désir de plaire à Dieu $"{ }^{32}$ Ce pieux souvenir de Dieu est l'une des manifestations de l'amour pour Dieu. «Cette disposition de l'âme est notre bonheur, et manifeste également la gloire de Dieu $» .^{33}$ Cette mémoire est possible à tout moment, et de différentes manières : par un geste, acte, pendant le travail ou le repos. Le travail ne dispense pas de l'obligation de la prière. Même au travail, on doit adorer Dieu, avec la bouche ou le cœur, par des psaumes, des hymnes et des cantiques spirituels, afin de remplir l'obligation de la prière pendant le travail. ${ }^{34}$

Lorsque vos mains sont occupées au travail, vous pouvez travailler la langue, si possible et de façon appropriée en vue de l'édification des croyants, ou tout au moins le cœur - pour adorer Dieu. ${ }^{35}$

Le souvenir constant de Dieu permet de faire croître l'amour pour Lui, et favorise l'accession à l'Amour parfait. De cette façon, l' homme lui-même excelle également.

Nous devons accomplir tous nos actes à la vue du Seigneur, et diriger vers Lui chacune de nos pensées comme s'il l'avait lui-même pénétrée. Vue sous cet angle, la crainte de Dieu peut produire de semblables actes et de pareils effets : ne pas supporter l'injustice, l'arrogance, l'orgueil et les routes menant au mal. C'est l'amour qui nous élève à la hauteur de la perfection. ${ }^{36}$

Le souvenir constant de Dieu est un trésor inestimable qui doit être protégé contre toute distraction. C'est un fil reliant la créature avec son Créateur. Il faut donc nous attacher à ce que ce fil ne se désagrège par notre faute, mais qu'il soit au contraire solide et durable. « Nous devrions garder soigneusement nos cœurs, ${ }^{37}$ pour que nos pensées sur Dieu ne se dispersent pas, ou qu'encore

30 M. Kanior, Historia monastycyzmu chrześcijańskiego, Kraków 1993, p. 92.

31 T. Spidlik, I. Gargano, Duchowość ojców greckich i wschodnich, p. 133.

32 T. Spidlik, I. Gargano, Duchowość ojców greckich i wschodnich, p. 133.

33 T. Spidlik, I. Gargano, Duchowość ojców greckich i wschodnich, p. 133.

${ }^{34}$ Cf. M. Kanior, Historia monastycyzmu chrześcijańskiego, p. 92.

${ }^{35}$ Reguły Dłuższe 37,2.

${ }^{36}$ Reguty Dłuższe 5,3.

37 Cf. Prz 4,23. 
elles ne soient perverties par toutes sortes de fausses affirmations au sujet de la mémoire de son miraculeux état. Au contraire, nous devons persévérer dans la sainte pensée de Dieu, par une mémoire incessante et pure, débarrassée en nos âmes de toute altération, tel un sceau indélébile. ${ }^{38}$ De telle façon que seul subsiste en nous un amour vivant pour Dieu, qui nous stimule à accomplir ses commandements et qui, protégé par ces derniers, devient solide et durable ${ }^{39}$

Basile lui-même était un homme de contemplation et de prière. ${ }^{40} \mathrm{La}$ contemplation est très importante pour l'homme, car à travers elle, on peut accéder à la beauté suprême : la beauté de la gloire de Dieu. ${ }^{41}$ Pour connaître la nature de la vraie beauté, la foi est nécessaire. Elle permet de même la contemplation. ${ }^{42}$ "L'homme, contemplant la beauté et attiré par elle de manière irrésistible, entre dans la vie en donnant ce qui est bon à autrui ». ${ }^{43}$

Une autre manifestation de l'amour pour Dieu consiste dans le travail continu sur soi-même, en une quête de perfection. La quête de la perfection par l' homme se poursuit tout au long de sa vie. L'amour pour Dieu exige un effort continu pour s'approfondir toujours plus.

Qui aime Dieu vraiment et profondément croit en la récompense promise par le Seigneur, ne se contente pas de ce qu'il a accompli, mais recherche à atteindre quelque chose de plus appréciable encore. Même s'il semble qu'il exécute une tâche excédant ses forces, il ne reste pas indifférent comme s'il avait accompli son devoir, mais s'efforce de progresser toujours, comme s'il avait l'intime conviction de n'avoir pas assez fait. ${ }^{44}$

Basile, ici, avait probablement à l'esprit que le progrès spirituel n'a jamais de limite, et peut ne jamais cesser tant que l'homme vit. Le progrès spirituel de l'homme conduit à la croissance de l'amour pour Dieu. Selon l'évêque de Césarée, la preuve fondamentale que l'homme aime Dieu réside dans son amour pour l'homme. Toutefois l'amour d'autrui, même envers ses proches (p. ex. parents), doit être subordonné à l'amour de Dieu. Malgré une telle

${ }^{38}$ Cf. Pnp 8,6.

39 Reguly Dłuższe 5,2.

${ }^{40}$ Cf. P. Kochanek, Kontemplacja $w$ dziełach Św. Bazylego, „Vox Patrum” 8 (1988), c. 15, p. 733.

\footnotetext{
${ }^{41}$ Cf. Homilia do psalmu 114.

42 Cf. Enarratio in Isaiam 5, 175, „Patrologia Graeca” 30, 412 C.

${ }^{43}$ P. Kochanek, Kontemplacja w dziełach Św. Bazylego, p. 730.

${ }^{44}$ Reguly Krótsze 121.
} 
attitude, Basile n'atténue en aucune manière l'importance de la famille et les liens existant en son sein. ${ }^{45}$ Les deux amours, l'amour de Dieu et du prochain, sont interdépendants et se renforcent mutuellement. Il est impossible d'aimer notre prochain sans aimer Dieu en même temps. A l'inverse, pas d'amour de Dieu sans amour du prochain.

Et pour bonne part, celui qui aime son prochain remplit le commandement de l'amour de Dieu, car Dieu reçoit cet amour comme un amour qui lui est aussi destiné. $^{46}$

Il semble que Basile considère l'accomplissement de la vocation comme un signe très important de l'amour pour Dieu. Chaque chrétien est doué d'une vocation. ${ }^{47}$ Le but de la vie de chaque chrétien est la gloire de Dieu, ${ }^{48}$ en imitation de l'amour du Christ pour son peuple et pour Dieu le Père. ${ }^{49} \mathrm{La}$ vocation invite plutôt le chrétien à " être en Christ ", elle est donc quelque chose de beaucoup plus profond qu'une simple imitation. "Être en Christ » signifie devenir membre du Corps du Christ par la réception du Baptême, de l'Eucharistie et de la Confirmation. ${ }^{50}$ Tout l'ascétisme et le mysticisme de Basile portaient les stigmates christologiques. ${ }^{51}$ Par conséquent, Basile encourage à suivre le Christ, à se parfaire dans le Christ. ${ }^{52}$

Tout doit être subordonné à la mise en ouvre de la vocation. Il n'y pas de chose plus importante que de suivre le Christ, ce qui est une expression de l'amour pour Lui. Toutes les choses de ce monde, y compris l'amour pour les proches, son moins importantes.

Si quelqu'un a un désir ardent de suivre le Christ, il ne devrait pas prendre soin de tout ce qui appartient à cette vie. Ni de l'amour pour les parents et les proches. ${ }^{53}$

${ }^{45}$ Cf. L. Małunowiczówna, Problem przyjaźni u Bazylego, Grzegorza Teologa i Jana Chryzostoma, „Roczniki Humanistyczne” 16 (1968), z. 3, p. 111.

${ }^{46}$ Reguły Dłuższe 3,2.

${ }^{47}$ Cf. Reguty Dłuższe 43,1.

${ }^{48}$ Cf. Reguły Dłuższe 20,2.

49 Cf. Reguly Dłuższe 43,1.

${ }^{50}$ Cf. J. Meyendorff, Teologia bizantyjska, p. 211.

${ }^{51}$ Cf. J. Górny, Wpływ św. Bazylego na rozwój życia monastycznego, „Vox Patrum” 2 (1982), z. 3 , p. 310 .

52 J. Górny, Wpływ św. Bazylego na rozwój życia monastycznego, p. 310.

${ }^{53}$ Reguty Dtuższe 8,2. 
Basile n'explique pas ici de quelle vocation il parle. Probablement il s'agit d'une vocation à la vie religieuse. Celui qui veut suivre le Christ doit changer radicalement sa vie, doit se libérer de ses attaches.

"Car celui qui veut suivre Dieu doit être libéré de tout rattachement à la vie, et cela ne peut se produire que par une rupture complète avec les vieilles habitudes et le fait de les oublier $»{ }^{54}$

Suivre le Christ n'est pas facile et simple. Cela exige beaucoup de sacrifices et d'abnégation. Basile explique la notion du sacrifice pour le Christ.

Elle implique la libération des liens qui nous emprisonnent dans cette vie terrestre et transitoire, l'indépendance par rapport aux engagements du monde, ce qui nous rend prêts et capables de prendre la route qui mène à Dieu. ${ }^{55}$

Le Cappadocien note à juste titre que, parfois, ce qui est terrestre éloigne l'homme du divin. Pour éviter cela, il est nécessaire de faire des sacrifices, des mortifications. Le départ volontaire de l'homme du monde et la vie dans la communauté est l'une des manifestations importantes de l'amour de l'homme pour Dieu. ${ }^{56}$ Il représente une expression d'une décision d'aimer Dieu en premier lieu, et de reporter les autres valeurs à leur juste place.

Un genre spécial de vocation est cité : prêcher la Parole de Dieu. Un des devoirs de celui qui a été appelé à proclamer la Parole est la préoccupation pour tous, l'amour et le dévouement à eux jusqu'à la mort. ${ }^{57}$ Basile, pour soutenir son avis, fait appel aux Saintes Écritures. ${ }^{58}$ Lamour pour Dieu doit être moteur pour proclamer la Parole de Dieu. Proclamation qui devrait avoir pour but la gloire de Dieu.

Il ne faut pas prêcher les enseignements pour se faire remarquer ou pour obtenir un profit, en essayant de se plier à l'auditoire ou pour satisfaire ses propres désirs ou besoins. Il faut être celui qui parle en présence de Dieu et pour sa gloire. ${ }^{59}$

Selon l'auteur de Reguty, le premier motif incitant à l'amour pour Dieu est le fait même de la création de l'homme par Dieu. L'amour de l'homme devrait

${ }^{54}$ Reguly Dluższe 5,2.

${ }^{55}$ Cf. Reguły Dłuższe 8,3.

${ }^{56}$ Cf. O. V. Desprez, Początki monastycyzmu. Dzieje monastycyzmu chrześcijańskiego do soboru efeskiego (431), Kraków 1999, p. 55.

57 Cf. Reguty Moralne 70,19.

${ }^{58}$ Cf. J 10,11; Dz 20,31; 1 Tes 2,9.

59 Reguly Moralne 70,23. 
être une réponse à l'amour du Créateur. La nature humaine est d'aimer ce qui est bon et beau. Dieu étant la plénitude, et même le critère fondamental de ces valeurs. En Dieu réside l'accomplissement de tous les désirs humains. Grâce à l'amour parfait de Dieu, l'homme est lui-même sanctifié. De plus, l'amour pour Dieu trouve une motivation dans la contemplation de l'amour de Dieu pour l'homme. L'amour pour Dieu est aussi une obligation édictée par Lui dans ses commandements.

Basile croit que la prière est une manifestation de l'amour de Dieu. Elle permet d'augmenter encore l'amour, et aussi à l'homme de se parfaire. La proclamation de la Parole de Dieu est un devoir pour tout chrétien, comme manifestation de l'amour envers Dieu. Un homme exprime aussi son amour pour Dieu par son travail et son effort dans l'amélioration de soi. La manifestation plus concrète de l'amour pour Dieu est l'amour d'autrui. Selon Basile, le fait d'imiter le Christ dans sa vie témoigne aussi de l'amour pour Dieu. Toute personne qui se rend compte de sa vocation témoigne de son amour pour Dieu. Une forme unique de mise en œuvre de l'amour pour Dieu est de quitter le monde et se vouer à la vie religieuse.

\section{Motifs et manifestations de la charité}

L'homme ne peut donner un témoignage véridique de son amour pour Dieu que par l'amour pour une autre personne. L'on ne peut pas prétendre aimer Dieu sans aimer son prochain. L'amour de l' homme trouve sa propre réalisation dans différentes situations de vie. L'amour agissant pour le prochain exige parfois effort, sacrifice et abnégation.

L'amour d'autrui est d'abord le premier commandement après l'amour pour Dieu. Un tel ordre a été établi par le Christ lui-même. ${ }^{60}$ L'existence même du commandement de l'amour du prochain fixé par Dieu est un motif suffisant d'obligation.

Basile le Grand, dans Reguły moralne, donne la réponse à la question de l'essence de l'amour du prochain. Aimer son prochain signifie « ne pas chercher à satisfaire son propre intérêt, mais l'intérêt de celui que l'on aime, ${ }^{61}$ pour le bien

\footnotetext{
${ }^{60}$ Cf. Reguły Dluższe 1 .

${ }^{61}$ Cf. 1 Kor 13,5.
} 
de son âme et de son corps ". ${ }^{62}$ Dans l'une des Reguly, à la question « comment pouvez-vous reconnaître que quelqu' un aime son frère, selon le commandement du Seigneur, et comment reconnaitre que quelqu'un ne l'aime pas de telle sorte", Basile répond : "L'amour a deux particularités : il est triste et troublé à cause de ce qui est préjudiciable à la personne aimée, et il se réjouit et se préoccupe de tout ce qui peut lui apporter des bienfaits $» .{ }^{63}$ L'amour du prochain est reconnu par le fait que l'on veut du bien pour lêtre aimé.

Lévêque de Césarée ne peut pas comprendre pourquoi les gens se soucient souvent plus des choses terrestres que de l'amour, qui ne se fane pas et qui a une valeur plus élevée que toute autre chose par ailleurs.

" Je suis surpris vraiment de voir que les gens ont tellement d'enthousiasme et de souci passionné pour ce qui passe et peut être détruit, et non pour les choses qui demeurent, en particulier la plus grande d'entre elles, l'amour, qui est la marque d'un chrétien $»{ }^{64}$

Basile a constamment protesté contre la mauvaise situation sociale, qui blessait l'amour chrétien du prochain. ${ }^{65} \mathrm{Il}$ comprenait parfaitement ce qu'est la pauvreté biblique. Il voyait la nécessité de partager les biens matériels avec les autres, il condamnait l'usure et l'enrichissement personnel au détriment des autres. ${ }^{66}$ L'enrichissement personnel d'un homme devrait servir à ceux qui sont dans le besoin. En ce sens, les mots de Basile sont importants et révolutionnaires : "C'est à celui qui a faim qu'appartient le pain que tu gardes, c'est à celui qui en a besoin que l'argent que tu as mis de côté appartient. En n'agissant pas ainsi, tu blesses autant que tu aurais pu soulager $»{ }^{67}$

Dans l'Homélie VI, 7 Basile le Grand prononce des mots très durs contre les riches : «Celui qui a faim doit recevoir le pain que tu caches; le nu, la robe dans ta garde-robe ; le déchaussé, les chaussures qui dépérissent chez toi ; le nécessiteux, l'argent que tu as enterré ». Ce n'est pas seulement celui qui est à l'origine du mal envers le prochain qui lui fait du mal, mais aussi celui qui ne lui fait pas de bien, refuse de l'aider.

\footnotetext{
${ }^{62}$ Reguly Moralne 80,22.

${ }^{63}$ Reguly Krótsze 175.

${ }^{64} \mathrm{O}$ wierze 5.

${ }^{65}$ Cf. K. Jasman, Był bardziej duszpasterzem i biskupem niż teologiem - św. Bazyli, „W Drodze" 7 (1979) nr 11, p. 56.

${ }^{66}$ Cf. Homilia 2 do psalmu 14; List 188.

${ }^{67}$ Cf. Jasman K. Był bardziej duszpasterzem i biskupem niż teologiem - św. Bazyli, p. 56.
} 
Le Cappadocien félicite ceux qui font un usage correct des biens terrestres: « $\mathrm{Si}$ quelqu' un comprend et utilise les biens temporels correctement, s'il redistribue les biens qui lui ont été donnés par Dieu, et ne les recueille pas pour son usage personnel et pour son propre plaisir, il est digne d'amour et d'honneur $»{ }^{68}$

L'homme doit partager les biens avec les autres, faute de quoi il leur fait du mal. C’est, en un sens, un motif négatif de la charité.

Le désir d'appartenir au groupe des disciples du Christ peut être un motif pour pratiquer la charité. ${ }^{69}$ La charité envers le prochain constitue un signe de cette appartenance. "A ceci tous connaîtront que vous êtes mes disciples, si vous vous aimez les uns les autres». ${ }^{70}$ Pour le chrétien, le modèle qui lui est donné est l'amour du Christ pour l' humanité. ${ }^{71}$ Un vrai chrétien doit chercher à conformer sa vie au Christ lui-même.

Les disciples du Christ se distinguent par le fait qu'ils aiment autrui pour lui-même. ${ }^{72}$ Malheureusement, il y a aussi des gens qui sont égoïstes, qui font du mal à leurs semblables, affaiblissant ainsi leur foi. Ces personnes peuvent difficilement être appelés chrétiens. ${ }^{73}$

Aimer son prochain est bien plus que simplement satisfaire aux commandements de Dieu. Pour Basile, il est très important que les motifs pour accomplir le commandement de l'amour soient nobles. Le motif principal de la charité est l'amour de Dieu. Il est parfaitement naturel pour un homme d'aimer une autre personne : « Rien n'est plus caractéristique de notre nature que d'entrer en relation avec d'autres, tout comme le soutien mutuel et l'amour du prochain $»{ }^{74}$

La volonté d'aimer son prochain d'une manière active revêt ainsi une importance particulière. L'auteur des Reguly croit que l'amour manifesté à l'homme est assimilable à l'amour pour Dieu. Pour l'amour qu'il aura manifesté à son prochain, l'homme recevra après la mort sa récompense : la vie dans le royaume des cieux. Par conséquent, les gens devraient rechercher toutes les occasions d'aider un autre être humain. Le Cappadocien croit qu'il « faut prendre soin et s'occuper du prochain avec zèle, réaffirmant que le Maître

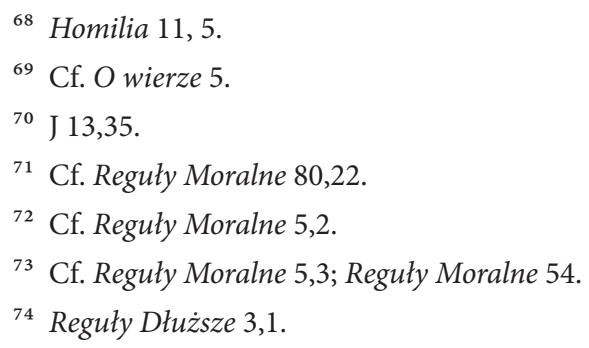


accueille, comme s'il lui était à lui-même destiné, tout acte de charité manifesté à autrui par ceux qui lui sont dévoués, leur promettant en retour le royaume des cieux $" .^{75}$

Il faut aimer et faire du bien à autrui, sans toutefois qu'il soit question que ce fût en vue d'une louange publique. Pour Dieu, il n'est de meilleurs actions que celles qui sont faites dans le secret, parce qu'assurément accomplies pour sa plus grande gloire. ${ }^{76}$ Tout homme est obligé à la charité. Personne ne vit pour soi-même, mais pour d'autres personnes. Toutes nos compétences, talents, grâces reçues, nous sont données pour servir les autres. Toute personne qui reçoit une grâce de Dieu doit la faire fructifier, et l'utiliser pour le bien et au profit d'autres personnes. Un homme doit être membre et médiateur de la grâce et la bonté de Dieu. ${ }^{77}$ "Puisque les dons de l'Esprit sont multiples, et que personne ne peut les posséder tous, et que tous ne peuvent pas posséder tel don particulier, tout le monde devrait raisonnablement et sincèrement se satisfaire de ce qu'il a reçu; tout le monde devrait vivre en accord avec soi-même dans l'amour du Christ, en tant que membre de son Corps; de même, celui qui a reçu moins de dons ne devrait pas jalouser celui qui le dépasse, pas plus que celui qui en a reçu davantage ne devrait mépriser le premier. Ceux qui sont divisés et sont en conflit les uns avec les autres méritent la destruction ». ${ }^{78}$

Ainsi, chaque homme est nécessaire, en tant que membre, au Corps mystique, et celui qui a reçu moins, par volonté divine, enrichit l'ensemble. Les hommes doivent se compléter, se recevoir mutuellement dans l'amour, ${ }^{79}$ et ne former qu'un seul Corps du Christ, uni dans et par l'amour qu' ils manifestent les uns pour les autres. Dans Reguła Moralna 60 Basile se réfère à quelques citations de la Bible, entre autres à la lettre de S. Paul aux Philippiens : « Ayez entre vous un même sentiment, un même amour, une même âme, une même pensée. Ne faites rien par esprit de parti ou par vaine gloire, mais que l' humilité vous fasse regarder les autres comme étant au-dessus de vous-mêmes. Que chacun de vous, au lieu de considérer ses propres intérêts, considère aussi ceux des autres ${ }^{80}$

\footnotetext{
75 Reguty Krótsze 207.

${ }^{76}$ Cf. Reguty Krótsze 276.

77 Cf. Reguty Krótsze 253.

78 Reguty Moralne 60.

${ }^{79}$ Cf. O wierze 1.

${ }^{80}$ Flp 2,2-4.
} 
Basile distingue deux motifs complètement opposés, par lesquels on atteint l'amour du prochain. Tout d'abord par crainte du jugement contre ceux qui défient le commandement du Seigneur, tel qu'exprimé en Jean : "Celui qui ne croit pas au Fils ne verra pas la vie, mais la colère de Dieu demeure sur lui ». ${ }^{81}$ Puis, par le désir de la vie éternelle, parce que: Son commandement est vie éternelle ${ }^{82}$ Seul l'amour qui tient compte de la vie éternelle est approprié.

L'évêque de Césarée explicite ainsi dans quelle mesure et de quel amour nous devons aimer notre prochain : «De l'amour qu' il vous a montré, et en ces mots : Aimez-vous comme je vous ai aimés. Il n'y a pas de plus grand amour que de donner sa vie pour ses amis. ${ }^{83}$ Mais si vous devez renoncer à votre propre vie, combien plus de dévouement devez-vous mettre dans les choses ordinaires, non pas pour satisfaire aux obligations humaines, mais pour plaire à Dieu, et en vue du bien de tous! $»{ }^{84}$

L'homme est appelé à aimer son prochain et à renoncer à sa vie pour luimême, parce que le Christ, lui aussi, est mort pour l'amour de tous, et que chaque chrétien est appelé à suivre son exemple. Cette situation est unique. Par conséquent, l'amour exprimé dans les petites choses de la vie devrait être quelque chose de naturel, d'évident.

L'amour pour celui qui nous fait du bien ne suffit pas. D'un vrai chrétien, il est exigé bien plus, parce que ceux qui ne croient pas en Lui n'agissent pas autrement. Dieu exige d'un chrétien qu'il aime même ceux qui le persécutent.

Les païens l'observent aussi, comme dit le Seigneur dans l'Évangile en disant: «Si vous aimez ceux qui vous aiment, quelle reconnaissance méritezvous ? Les pécheurs aussi aiment ceux qui les aiment $»{ }^{85} \mathrm{Si}$ le prochain nous fait du mal, même dans ce cas nous devons l'aimer, non seulement à cause du commandement, mais aussi parce qu'il devient la cause des biens promis par le Seigneur : «Heureux serez-vous, lorsqu'on vous outragera, qu'on vous persécutera et qu'on dira faussement de vous toute sorte de mal, à cause de moi. Réjouissez-vous et soyez dans l'allégresse, parce que votre récompense sera grande dans les cieux! ». ${ }^{86}$

\footnotetext{
$81 \mathrm{~J} \mathrm{3,36.}$

${ }^{82}$ Reguly Krótsze 163.

${ }^{83} \mathrm{~J} 15,12$.

${ }^{84}$ Reguły Krótsze 162.

${ }^{85}$ Łk 6,32.

${ }^{86}$ Reguly Krótsze 163.
} 
Il faut aimer ses ennemis à cause du commandement de Dieu. Cet amour est aussi le résultat de la confiance en la Parole de Dieu qui bénit ceux qui aiment leurs ennemis et promet " une grande récompense dans les cieux ». Paradoxalement, l' homme qui se montre hostile au chrétien s'avère être source de bénédiction : "Ce sont nos ennemis qui nous conduisent à la bénédiction dont parle le Seigneur ${ }^{87}$

L'amour est une occasion de se soigner du péché. Cependant, seule une personne qui « aime sincèrement » peut bénéficier de ce remède. ${ }^{88}$ Sans aucun doute l'amour possède-t-il une valeur pédagogique. Il permet d'éduquer et de façonner l'homme correctement. Pour cautionner son point de vue, le saint évêque de Cappadoce cite le Livre des Proverbes : "Celui qui aime vraiment éduque attentivement $» .{ }^{89}$

Pour le Père Cappadocien, aimer son prochain, c'est lui vouloir du bien. On l'a dit : le motif principal de l'amour du prochain est l'amour de Dieu. Celui qui veut être un disciple du Christ doit aussi aimer autrui. L'existence même d'un commandement de l'amour donné par Dieu devrait inciter l'homme à le suivre. Basile parle aussi des motifs de l'amour du prochain que sont la crainte du jugement de Dieu et la volonté de vivre éternellement. Pour un chrétien, c'est bien le deuxième motif qui est approprié. De même, le désir d'imiter la vie du Christ est un motif supplémentaire de pratiquer la charité. Jésus aimait du même amour ses amis et ses ennemis. Le Christ étant, selon l'évangéliste Jean, la Parole de Dieu incarnée, il va donc de soi que vivre selon cette Parole, qui appelle à l'amour, doit être le motif même de l'amour.

L'amitié est l'une des nombreuses manifestations de l'amour du prochain citées par le Cappadocien. La base de l'amitié est la foi commune. ${ }^{90}$ Basile voit dans l'amitié certains biens spirituels. De l'amitié découlent la joie, le soutien mutuel dans les temps difficiles, l'aide et l'incitation à faire le bien. Pour que cela se passe ainsi, l'amitié doit être subordonnée à l'amour de Dieu. ${ }^{91}$ Basile lui-même aimait à cultiver l'amitié. Dans l'une de ses lettres, il a écrit à propos de ses amis: «J'ai donné beaucoup d'amour, de ma prime jeunesse à ma vieillesse

\footnotetext{
${ }^{87}$ Reguly Krótsze 176.

${ }^{88}$ Cf. Reguly Dluższe 7,1.

${ }^{89} \operatorname{Prz} 13,24$.

90 Cf. List 133; List 154.

${ }^{91}$ L. Małunowiczówna, Problem przyjaźni u Bazylego, Grzegorza Teologa i Jana Chryzostoma, p. 110.
} 
actuelle ${ }^{92}{ }^{92}$ Lamour est ce qui unit des amis, ce qui crée les liens entre les êtres. Il est donc, pour Basile, d'une valeur immense. ${ }^{93}$ " Le bien le plus précieux de tous est, à nos yeux, dans l'amour qui nous lie avec vous ". ${ }^{94}$

Quand un homme aime son prochain, il devient alors comme un instrument dans les mains de Dieu, avec l'aide duquel son amour et sa miséricorde atteignent les nécessiteux. Le partage des biens matériels avec les pauvres est normal et nécessaire. Un homme devrait aider son prochain, travailler pour lui, « afin que Dieu puisse donner aux frères faibles ce qui est nécessaire pour eux $» .^{95}$ Le motif du travail n'est pas de satisfaire un auto-amour, mais l'amour du prochain. ${ }^{96}$ Le but du travail pour Basile est d'aider notre prochain. L'homme ne travaille donc pas principalement pour lui-même, mais pour que ceux qui en ont besoin, les plus pauvres, aient l'opportunité de vivre des fruits de ce travail. «Celui qui le peut devrait travailler pour être en mesure d'apporter de l'aide aux nécessiteux $» .^{97}$

L'homme qui vise l'excellence devrait soutenir les pauvres au moyen des fruits de son travail : «Une caractéristique de celui qui vise l'excellence est de travailler jour et nuit pour avoir quelque chose à partager avec ceux qui en ont besoin ${ }^{98}{ }^{98}$

Dans Reguła Moralna 48:7, Saint Basile soutient son avis en citant la Lettre aux Ephésiens : "Que le voleur cesse de voler. Qu'il prenne plutôt le peine de travailler honnêtement pour avoir de quoi partager avec ceux qui sont dans le besoin $» .{ }^{99}$ Le sens du travail, pour Basile, réside dans le fait que ses fruits aident les pauvres, les faibles, les malades : « L'objectif qui doit guider chacun dans son travail doit être d'aider les pauvres, et non pas de satisfaire à ses propres besoins. Ainsi, nous éviterons les seules revendications pour nous-mêmes, et nous serons bénis par le Seigneur pour avoir prodigué de l’amour à nos frères,

92 List 272, 1.3 do Sofroniosa.

${ }^{93}$ Cf. L. Małunowiczówna, Problem przyjaźni u Bazylego, Grzegorza Teologa i Jana Chryzostoma, p. 115.

${ }^{94}$ List 203,2.

95 Reguły Dłuższe 37,1.

${ }^{96}$ Cf. J. Gliściński, Zagadnienia społeczne u Ojców Kościoła, „Collectanea Theologica” 63 (1993) nr 1, p. 58.

97 Reguly Moralne 48,7.

98 Reguly Dłuższe 42,1.

99 Ef $4,28$. 
car il a dit : « À chaque fois que vous l'avez fait à l'un de ces plus petits qui sont mes frères, c'est à moi que vous l'avez fait ». ${ }^{100}$

On peut en conclure que le travail pour les autres est comme une médecine qui protège de l'égoïsme, et qu'il contribue à s'ouvrir à d'autres personnes et à Dieu. Cette compréhension de la signification du travail humain est certainement unique et caractéristique de Saint Basile, en ce sens qu'à ses yeux, l'aide au prochain ne doit pas seulement provenir de notre surplus. Une telle position est inconnue chez les autres Pères de l'Église qui lui sont contemporains : «Au cour de la réflexion de Basile sur la question des biens, il y a une vision biblique proclamant que "tout ce que l' homme possède est don de Dieu", ${ }^{101}$ et n'appartient pas à l'homme uniquement. Les biens dont l'homme dispose sur terre lui sont donnés par le Créateur lui-même. ${ }^{102}$ L'homme ne possède rien qui ne lui soit préalablement donné par Dieu. Les enseignements de Basile en termes de biens matériels "sont basés sur la subordination de la propriété privée aux devoirs de la charité chrétienne. Chacun est seul propriétaire de ses biens à concurrence du nécessaire, et aussi longtemps qu'il n'y a pas besoin de les prêter à qui en aurait besoin $»{ }^{103}$

Le fait de partager de l'amour peut paradoxalement avoir pour effet que cet amour soit multiplié. Basile compare la situation au fait de donner de l'argent aux banquiers pour plus encore de bénéfices ultérieurs : «Alors si on pratique le fait de donner de l'argent aux banquiers pour en retirer un profit (...) il est donc nécessaire que le bénéficiaire de la grâce en fasse de même bénéficier son prochain.». ${ }^{104}$

D'autre part, quand quelqu'un garde pour lui-même un cadeau ou une grâce, il séloigne du plan de Dieu : "Celui qui garde pour lui-même un don de la grâce de Dieu, et ne l'utilise pas pour le bien des autres, se rend coupable comme l' homme qui a caché son talent $» .^{105}$

${ }^{100}$ Reguty Dłuższe 42,1.

${ }^{101}$ L. Andrzejewski, Własność prywatna i jej ograniczenia według św. Bazylego, „Ateneum Kapłańskie” 16 (1930), t. 26, z. 5, p. 455.

102 Cf. R. Andrzejewski, Myśl społeczna św. Bazylego Wielkiego w nauce św. Ambrożego, „Roczniki Teologiczno-Kanoniczne”, t. XXVII (1980), z. 4, p. 180.

${ }^{103}$ L. Andrzejewski, Własność prywatna i jej ograniczenia według św. Bazylego, p. 455.

${ }^{104}$ Reguly Krótsze 254.

${ }^{105}$ Reguly Krótsze 62. 
Un tel comportement n'est pas digne d'un chrétien, qui s'exposerait à la punition de Dieu, parce que tous les dons qui nous sont faits le sont aussi pour le bien des autres. Dieu donne à l'homme la capacité d'aimer les autres, et Il veut que nous en usions : "Donc si le Seigneur nous a donné des graines, il est clair qu'il exigera la moisson $»{ }^{106}$

En raison de l'amour du Christ, il n'est pas normal pour un homme de n'avoir à l'esprit que ses propres avantages. ${ }^{107}$ Lamour pour le Christ implique donc l'amour du prochain, et réciproquement. La charité doit se manifester dans l'aide matérielle à autrui et, « conformément à la volonté de Dieu, il faut attacher grand soin à ce dont le prochain a besoin $» .{ }^{108}$ Laide au prochain est non seulement une question de choix, mais elle est la " volonté de Dieu », elle est ce que Dieu exige de nous : en tant que disciples du Christ, notre devoir est de nous soutenir de toutes les manières possibles et, si cela s'avère nécessaire, d'aller jusqu'à donner notre propre vie pour notre prochain, comme le Christ l'a lui-même fait. ${ }^{109}$

L'auteur de Reguly aborde également l'un des problèmes actuels de son temps : l'esclavage. Bien qu' il ne soit évidemment pas opposé à cette pratique, il précise cependant ce que devrait être la relation entre maître et esclave, en soulignant plutôt, et de manière vigoureuse, la nécessité d'un service mutuel et de relations amicales. Basile appelle à traiter les esclaves à l'imitation du Christ. Le Père Cappadocien, même de manière non explicite, encourage à l'amour pour les esclaves. L'Évêque de Césarée les désigne comme «frères dans le Christ qui, comme quiconque, ont droit à la dignité et le respect de son peuple ${ }^{110}$ Basile nous rappelle que les maîtres doivent se rappeler qui est le seul et vrai Seigneur : " Ils devraient fournir aux esclaves autant qu' ils reçoivent de leur part; ils devraient agir à leur égard avec la crainte de Dieu, et avec douceur, suivant l'exemple du Seigneur $»{ }^{111}$ Christ doit être le vrai Seigneur d'un esclave, tout comme il l'est de son propriétaire. Il est le modèle de l'amour du prochain. Il ne fait pas de distinction entre les gens, qu' ils soient libres ou

\footnotetext{
106 Reguty Dłuższe 3,1.

107 Cf. Reguty Dłuższe 7,1.

108 Reguty Moralne 48,6.

109 Cf. Reguly Krótsze 181.

110 P. Kochanek, Nauczanie społeczne Bazylego wielkiego. Wybrane, „Roczniki Teologiczno--Kanoniczne" 36 (1989) z. 4, p. 122.

111 Reguly Moralne 75,2.
} 
esclaves. Il est venu pour sauver chacun, indépendamment de son statut et de son origine ${ }^{112}$. En justification de son point de vue sur l'esclavage, l'évêque de Césarée se réfère aux sources bibliques. Il cite deux passages : l'Évangile selon saint Jean : «Vous m'appelez Maître et Seigneur; et vous dites bien, car je le suis. Si donc je vous ai lavé les pieds, moi, le Seigneur et le Maître, vous devez aussi vous laver les pieds les uns aux autres; car je vous ai donné un exemple, afin que vous fassiez comme je vous ai fait ${ }^{113}$ et l'Epitre aux Ephésiens : «Et vous, maîtres, agissez de même à leur égard, et abstenez-vous de menaces, sachant que leur maître et le vôtre est dans les cieux, et que devant lui il n'y a point d'exception de personnes $» .{ }^{114}$

L'amour mutuel est le fondement des liens familiaux. Les parents sont les premiers à en être dotés. "Par nature » l' homme est porté à aimer davantage ceux qui lui font du bien. Ici, on peut parler d'un engagement envers la personne qui fait ce bien. ${ }^{115}$ Les liens qui unissent parents et enfants illustrant probablement au mieux un tel attachement. Les parents sont les premiers qui, les aimant, font du bien à leurs enfants. Les parents sont ceux qui, en premier lieu, dispensent l'amour à leurs enfants, en leur transmettant la vie. Cela n'est pas si évident, car même dans les temps qui sont ceux de Basile, les cas d'avortement et d'abandon de nouveau-nés sont légion. Il s'oppose par ailleurs avec force à de telles pratiques, ${ }^{116}$ qualifiant ouvertement « d'assassinat la destruction du fotus et l'abandon des nourrissons $» .{ }^{117}$ Les enfants, quant à eux, doivent « honorer leurs parents et leur obéir en ce qui n'est pas contraire aux commandements de Dieu $»{ }^{118}$ Basile décrit également ici les limites de l'obéissance aux parents : la conformité aux commandements de Dieu. Le Cappadocien rappelle saint Paul dans la Lettre aux Ephésiens : " Enfants, obéissez à vos parents, selon le Seigneur, car cela est juste. Honore ton père et ta mère - le premier commandement qui soit assorti d'une promesse - afin

112 Reguly Krótsze 245.

113 J 13,13-15.

114 Ef 6,9.

115 Cf. Reguty Dłuższe 2,2.

${ }^{116}$ S. Longosz, Rodzina wczesnochrześcijańska i jej zadania w nauczaniu św. Bazylego, „Roczniki Teologiczno-Kanoniczne” 28 (1981), z. 4, p. 158.

117 S. Longosz, Rodzina wczesnochrześcijańska i jej zadania w nauczaniu św. Bazylego, p. 158.

${ }^{118}$ Reguly Moralne 76,1. 
que tu sois heureux et que tu vives longtemps sur la terre $» .{ }^{119}$ Basile assimile l'amour fraternel à l'atmosphère familiale. Ainsi appelle-t-il la mère reine de la maison, qui nourrit et qui prend soin de tous. ${ }^{120}$ Les parents devraient plutôt éduquer leurs enfants avec douceur et patience, dans la discipline et la connaissance du Seigneur, en ne leur donnant jamais - pour autant que cela dépende d'eux - quelque raison de colère et de tristesse. ${ }^{121}$

Saint Basile fait ici référence aux deux textes de l'Apôtre des nations. Dans la Lettre aux Ephésiens : «Et vous, pères, n’irritez pas vos enfants, mais élevez-les en les corrigeant et en les instruisant selon le Seigneur ${ }^{122}$ et dans la Lettre aux Colossiens : « Pères, n'irritez pas vos enfants, de peur qu' ils ne se découragent ${ }^{123}$ Dans l'éducation de leur progéniture, les parents doivent tendre vers l'intérêt supérieur de l'enfant. Ils devraient donc élever un enfant de façon à être en mesure d'atteindre leur but ultime : la vie éternelle. ${ }^{124}$

Aimer son prochain se manifeste également dans la prière. Celle des parents pour les enfants est une expression de l'amour parental. Lamour des parents « ne devrait pas se limiter aux mots, à leur naissance et à l'assurance de bonnes conditions matérielles, ce qui est dans l'ordre des choses, mais il devrait être aussi caractérisé par un caractère surnaturel, dirigé vers Dieu, de manière consciente, volontaire et concomitante à la prière fréquente pour les enfants $»{ }^{125}$ Le Cappadocien en parle à un certain père dans l'une de ses lettres : «Tu dois démontrer l'amour pour les enfants non seulement en paroles, comme tu l'as déjà fait depuis que tu es devenu père. Tu dois les aimer non seulement de cet amour naturel qui, comme tu l'as toi-même déjà dit, est aussi le fait des créatures dépourvues de raison, mais tu dois essayer de les aimer d'un amour conscient et volontaire, conscient que tu es qu' ils sont dignes des prières de leur père ». ${ }^{126}$

Il est indubitable que Basile a foi en l'immense puissance de la prière. Il recommande de prier pour le perfectionnement spirituel, et de remercier Dieu

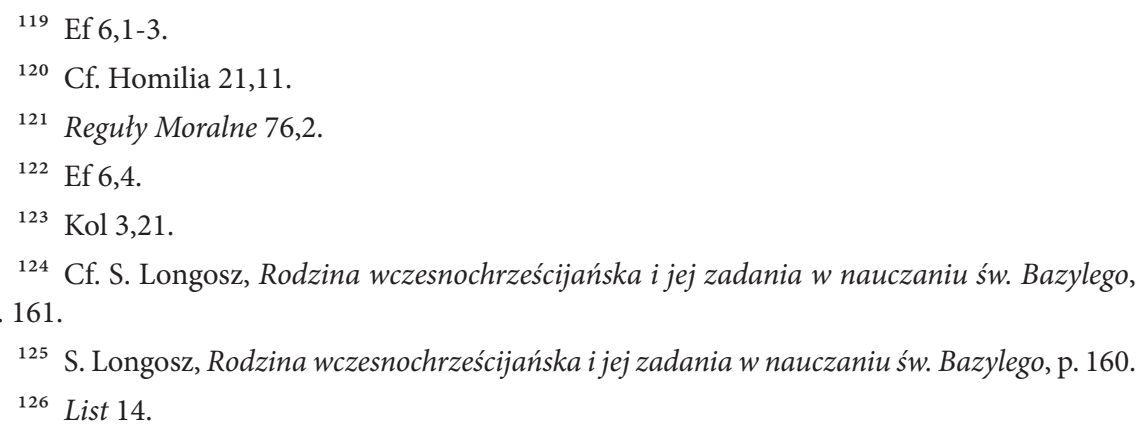


pour les prochains. ${ }^{127}$ Le chrétien doit également s'attacher, dans la mesure du possible, à ceux qui font du mal. ${ }^{128}$ En aucun cas il ne faut se venger d'une personne qui a fait quelque chose de mal. Une telle conduite ne se justifie par rien. ${ }^{129}$

Basile distingue deux sortes d'amour de l'ennemi. La première est l'amour de son âme, qui consiste à réprimander l'ennemi, et tout faire pour amener ce dernier à la repentance. La deuxième étant l'amour de l'ennemi contre son corps, que nous lui montrons en lui prêtant ce dont il a besoin pour vivre. ${ }^{130}$ Tout chrétien se voit appelé à la pratique de l'une comme de l'autre.

L'amour pour l'ennemi comme celui pour l'ami n'a pas de limite. ${ }^{131}$ « Nous devrions montrer l'amour jusqu'à la mort aux pécheurs ainsi qu'aux justes. Car il est dit: Dieu montre son amour, en ce que Christ est mort pour nous, alors que nous étions encore pécheurs $»{ }^{132}$

L'amour pour les ennemis doit suivre le modèle de l'amour parfait du Christ pour son peuple. Il est également mort pour les pécheurs, il les a rachetés.

Prier pour eux est un moyen de manifester de l'amour pour ennemis. ${ }^{133} \mathrm{Le}$ Père de Cappadoce se réfère à un fragment de l'Évangile selon saint Mattieu : "Aimez vos ennemis, bénissez ceux qui vous maudissent, faites du bien à ceux qui vous haïssent, et priez pour ceux qui vous maltraitent et qui vous persécutent, afin que vous soyez fils de votre Père qui est dans les cieux ». ${ }^{134}$

Nous devrions faire la preuve de notre générosité en faisant d'élever vers Dieu, d'un cœur pur, notre prière pour la personne qui a fait du mal, et dire : Seigneur, pardonne-lui ce péché. ${ }^{135}$ Ainsi, nous ne deviendrons pas coupables de jugement, comme l'est celui qui est en colère contre son frère. ${ }^{136}$ En même temps il faut réprimander l'auteur du dommage, et le convaincre de se préserver de la colère

\footnotetext{
127 Cf. Reguly Moralne 56,5 i 70,14.

128 Cf. Reguty Moralne 5,4.

129 Cf. Reguty Moralne 49,3.

${ }^{130}$ Cf. Reguly Krótsze 176.

${ }^{131}$ Cf. Rz 5,8.

${ }^{132}$ Reguly Krótsze 5,8.

${ }^{133}$ Cf. Reguty Moralne 56,6.

134 Mt 5,44.

135 Cf. Dz 7,60.

${ }^{136}$ Cf. Mt 5,22.
} 
qui touche ceux qui désobéissent. ${ }^{137}$ Celui qui néglige cela et ignore un péché par générosité, pèche doublement. Il transgresse un commandement qui dit: « Tu auras soin de reprendre ton prochain, mais tu ne te chargeras point d'un péché à cause de lui », ${ }^{138}$ car par ton silence tu deviens un pécheur complice. ${ }^{139}$

À part la prière il faut s'assurer que le prochain ne commette pas d'autres péchés. Si nécessaire, il faut l'avertir. Regarder passivement un frère pécheur, c> est aussi pécher. "Il ne faut pas rester indifférent à ceux qui pèchent », ${ }^{140} \mathrm{ni}$ " garder le silence face au péché de quelqu'un ». ${ }^{141}$

Le Père Cappadocien appelle à l'amour du prochain qui, potentiellement, " peut même affliger si c'est à son avantage ». ${ }^{142}$ Un tel amour est modelé sur l'amour du Christ. A titre d'exemple, l'auteur de Reguly met en exergue S. Paul et son amour pour les premières communautés ecclésiales. ${ }^{143}$

Si vous voyez le péché, il faut amener son auteur à en prendre conscience. C'est aussi une manifestation de l'amour du prochain. $S$ ' il regrette son acte, il faut lui pardonner. Il est nécessaire de réagir contre le mal, le péché - condamnable et d'essayer de convertir le pécheur. N’opposer aucune réponse au Mal constitue de fait à soi-même pécher.

Le chrétien devrait pardonner, et s'efforcer de ne pas se souvenir des dommages subis. ${ }^{144}$ De cette manière, non seulement il prouve sa capacité à l'amour mais, plus important, il accomplit les commandements de Dieu. Ce qui est aussi une façon de leur montrer son amour. Lévêque de Cappadoce se réfère ici à l'Évangile selon saint Mathieu : "Si vous pardonnez aux hommes leurs offenses, de même votre Père céleste vous pardonnera. Mais si vous ne pardonnez pas aux hommes, votre Père non plus ne vous pardonnera pas ». ${ }^{145}$ Le pardon pour les coupables est une condition préalable pour obtenir le pardon de Dieu.

\footnotetext{
137 Cf. 1 Tes 1,10; Ef 5,6.

138 Kpł 19,17.

139 Reguly Krótsze 232.

140 Reguly Moralne 52,1.

${ }^{141}$ Reguty Moralne 52,2.

142 Reguty Moralne 5,5.

143 Cf. Łk 17,3; Ef 5,11.

${ }^{144}$ Cf. Reguly Moralne 3.

145 Mt 6,14.
} 
Basile permet de "se détourner du pécheur ", mais à la seule condition d'avoir fait tout ce qui était possible pour sa conversion. ${ }^{146}$ Une telle situation est possible, mais uniquement en dernier recours. Auteur de Reguły, pour appuyer son point de vue, cite ce passage de l'Évangile selon saint Matthieu : "Si ton frère a péché (contre vous), va et reprends-le seul à seul. S’ il écoute, tu as gagné ton frère. Mais, s'il ne t'écoute pas, prends avec toi une ou deux personnes, afin que toute l'affaire se règle sur la déclaration de deux ou de trois témoins. $S^{\prime}$ il refuse de les écouter, dis-le à l'Église; et s'il refuse aussi d'écouter l'Église, qu'il soit pour toi comme un païen et un publicain $» .{ }^{147}$

L'amour pour les ennemis n'est de loin pas facile à mettre en œuvre, mais il est néanmoins possible : «Dieu, qui est bon et juste, ne nous imposerait pas un tel commandement s'il était impossible de le suivre. Il a également démontré qu'il s'agit d'une nécessité, imposée par la nature même ». ${ }^{148}$ Dieu n'exigerait jamais de l'homme plus que celui-ci ne pourrait donner. En intimant à l'homme le commandement d'aimer ses ennemis, Il lui donne en même temps la faculté d'accomplir cet acte. Car l'amour des amis est naturel, il est imprimé dans la nature humaine elle-même. En revanche, aimer ses ennemis exige de l' homme un effort certain.

Une manifestation concrète de la charité chrétienne consiste en la volonté de dissuader le prochain du péché et du mal. Et ceci demande prudence, sagesse, persévérance, en même temps qu'une perception adéquate de la situation : "Comme autrefois le serpent qui, se parant d'une apparence séduisante, sut approcher l'homme, et lui parla de telle façon qu'il se détournât de Dieu et tombât dans le péché, ${ }^{149}$ de même devons-nous privilégier la bonne attitude, la bonne manière et le bon moment, et choisir les mots justes aux fins de détourner les gens de péché, ${ }^{150}$ et les conduire à Dieu. En de telles expériences, nous devons veiller à la persévérance, ainsi qu'il est écrit... », ${ }^{151}$ jusqu'à la fin. ${ }^{152}$

Par conséquent, le chrétien a le devoir de réparer tout dommage causé par Satan par l'entremise du péché. Pour dissuader le prochain d'accomplir un acte

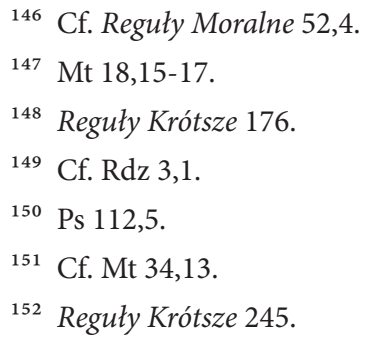


mauvais, on peut user même de l'agilité et de la ruse dont fit autrefois preuve le serpent pour tromper les premiers humains. En prenant soin cependant que ladite la ruse agisse au service du Bien.

Basile considère comme un signe de l'amour du prochain le soin que l'on apportera au corps et à l'âme des autres, à savoir tient pour obligation de fournir les biens nécessaires à l'organisme de son prochain. Plus pauvre et vulnérable est le prochain, plus grande est cette obligation. Basile donne comme but au travail humain l'aide envers les pauvres et les nécessiteux. De même que la prière aux intentions du prochain manifeste l'amour qu'on lui porte. L'amour du prochain doit concerner l'homme en son entier, corps et âme. Ainsi, nulle dispense dans la prière pour ses ennemis. L'amour du prochain exige parfois de répondre au péché, au Mal. Ce doit être une réaction ferme, parfois douloureuse pour le pécheur, mais pour son bien et sa conversion. On l'a dit plus haut, la question de l'esclavage est importante aux yeux de Basile. Il appelle chacun au même amour, tant pour les esclaves que pour tout autre être humain car pour lui, il n'existe qu'une seul Maître, pour les esclaves comme pour les hommes libres : Jésus-Christ.

L'enseignement de l'amour du prochain chez Basile n'est pas seulement une théorie. L'Évêque de Césarée le confirme par sa vie. Alors qu'il est encore prêtre, il organise à Césarée des repas gratuits. Et pour ce faire, il vend sa propriété. ${ }^{153}$ Bien que Basile soit considéré principalement comme un moraliste, « sa doctrine morale est en grande partie le reflet de sa sainteté personnelle », ${ }^{154}$ manifestée au long d'une vie faite de prière, d'ascétisme et de bienfaisance à grande échelle, accordant la plus grande considération à la vie de ses communautés et de toute l'Église, ${ }^{155}$ n'ayant de cesse de mettre en exergue, dans son enseignement moral, l'ascétisme pourtant actif d'un vie religieuse qu'il avait choisie par la seule exigence de l'amour, ${ }^{156}$ distribuant ses richesses aux pauvres et limitant au minimum ses propres besoins. ${ }^{157}$ Pour un tel amour et un tel dévouement à son endroit, le peuple de Césarée lui rendra la pareille. ${ }^{158}$

${ }^{153}$ Cf. J. Naumowicz, Instytucje charytatywne św. Bazylego. Bazyliada, „Vox Patrum” 16 (1996) z. 30-31, p. 126.

\footnotetext{
154 J. Pryszmont, Patrystyczna myśl moralna, p. 132.

155 Cf. J. Naumowicz, Instytucje charytatywne św. Bazylego. Bazyliada, p. 138.

156 Cf. J. Czuj, Patrologia, Poznań 1954, p. 151.

157 J. Pryszmont, Patrystyczna myśl moralna, p. 132, 133.

${ }^{158}$ Cf. H. Campenhausen, Ojcowie Kościoła. Greccy Ojcowie Kościoła, Warszawa 1967, p. 93.
} 


\section{La fin}

L'analyse des textes de Saint Basile réalisée dans cet article sur la motivation de l'amour de Dieu et du prochain nous permet de dégager les conclusions suivantes.

L'amour de l'homme pour Dieu est un postulat de la nature réflexive humaine. Ce motif découle du fait que l'homme fut créé par Dieu, à son image et ressemblance.

Saint Basile le Grand nous enseigne que l'amour de Dieu est la résultante pour l'homme du désir de sa vérité, de sa bonté et de sa beauté.

Selon l'enseignement de saint Basile, ces valeurs permettent en fin de compte à l'homme de trouver Dieu.

La motivation de considérer Dieu comme amour suprême est une dimension sotériologique de cet amour, qui se révèle au monde dans le Christ, seul Sauveur de l' homme qui, avec son Père du Ciel, nous envoie le Saint-Esprit, qui renouvelle constamment la face de la terre.

Selon l'évêque Cappadocien, l'amour pour Dieu peut aussi être motivé par une crainte de Dieu résultant du cœur et de l'esprit humains, alors qu'il devrait découler de la reconnaissance pour les dons reçus de Lui, et en premier lieu pour le don de la création.

Basile expose que la réponse humaine à l'amour de Dieu devrait être une relation cordiale avec lui, nourrie par la prière, la contemplation, le travail et la prédication de la Parole, de même que par le respect de ses commandements.

Le motif ultime de cet amour et sa manifestation trouvent leur réalisation, en tant qu'idéal de toute vie chrétienne, dans l'imitation de la vie du Christ.

Lamour de l' homme pour Dieu se vérifie, selon ce que Basile enseigne, dans l'amour de l'homme pour l'homme qui prend source dans le commandement de Dieu « tu aimeras ton prochain comme toi-même».

Basile considère que la capacité de l'homme à se réjouir avec ceux qui se réjouissent, et de souffrir avec ceux qui souffrent, est un critère de distinction de cet amour.

La vérification de ce genre d'amour est l'amour des ennemis, l'aide aux pauvres et aux malades, en conformité avec les principes formulés par Basile: "Ce n'est pas seulement celui qui est à l'origine du mal à son prochain qui lui fait du mal, mais aussi celui qui ne lui fait pas de bien, refuse de l'aider ».

Ce principe a été illustré par les actes concrets et systématiques d'aide spirituelle et matérielle aux pauvres et aux malades, particulièrement au travers du complexe de chambres construit par S. Basile, appelé « Basiliade». 


\section{Bibliography}

Andrzejewski L., Własność prywatna i jej ograniczenia według św. Bazylego, „Ateneum Kapłańskie" 16 (1930), t. 26, p. 433-456.

Andrzejewski R., Myśl społeczna św. Bazylego Wielkiego w nauce św. Ambrożego, „Roczniki Teologiczno-Kanoniczne” XXVII (1980), z. 4, p. 212-232.

Bazyli Wielki, Homilie i kazania, Kraków 1947.

Bazyli Wielki, Listy, Warszawa 1972.

Bazyli Wielki, O wierze, in: „Źródła monastyczne”, Bazyli Wielki, Pisma ascetyczne, Kraków 1995, t. 1, p. 82-95.

Bazyli Wielki, Reguły Dłuższe, in: „Źródła monastyczne”, Bazyli Wielki, Pisma ascetyczne, Kraków 1995, t. 2, p. 37-181.

Bazyli Wielki, Reguły Krótsze, in: „Źródła monastyczne”, Bazyli Wielki, Pisma ascetyczne, Kraków 1995, t. 2, p. 185-453.

Bazyli Wielki, Reguły Moralne, in: „Źródła monastyczne”, Bazyli Wielki, Pisma ascetyczne, Kraków 1995, t. 1, p. 99-269.

Campenhausen H., Ojcowie Kościoła. Greccy Ojcowie Kościoła, Warszawa 1967.

Czuj J., Patrologia, Poznań 1954.

Desprez V., Początki monastycyzmu. Dzieje monastycyzmu chrześcijańskiego do soboru efeskiego (431), Kraków 1990.

Enarratio in Isaiam 5, 175, „Patrologia Gracea” 30, 412 C.

Gliściński J., Zagadnienia społeczne w pismach Ojców Kościoła, „Collectanea Theologica” 63 (1993) nr 1, p. 53-65.

Górny J., Wpływ św. Bazylego na rozwój życia monastycznego, „Vox Patrum” 2 (1982), z. 3, p. 298-312.

Homilia in divites 6, „Patrologia Gracea” 31, 296 C.

Hryniewicz W., Człowiek w mocy Ducha Świętego. Zarys pneumatologii Ojców Wschodnich, „Znak” 29 (1977), p. 783-792.

Jasman K. Był bardziej duszpasterzem i biskupem niż teologiem - św. Bazyli, „W Drodze” 7 (1979) nr 11, p. 51-58.

Kanior M., Historia monastycyzmu, Kraków 1993.

Kochanek P., Kontemplacja w dziełach św. Bazylego, „Vox Patrum” 8 (1988), z. 15, p. 717-734.

Kochanek P., Nauczanie społeczne Bazylego Wielkiego. Zagadnienia wybrane, „Roczniki Teologiczno-Kanoniczne" 36 (1989), z. 4, p. 119-131.

Longosz S., Rodzina wczesnochrześcijańska i jej zadania w nauczaniu św. Bazylego, „Roczniki Teologiczno-Kanoniczne” 28 (1981), z. 4, p. 149-168.

Małunowiczówna L., Problem przyjaźni u Bazylego, Grzegorza Teologa i Jana Chryzostoma, „Roczniki Humanistyczne” 16 (1968), z. 3, p. 107-132.

Meyendorff J., Teologia bizantyjska, Warszawa 1984. 
Naumowicz J., Instytucje charytatywneśw. Bazylego Wielkiego. Bazyliada, „Vox Patrum” 16 (1996) z. 30-31, p. 125-139.

Pryszmont J., Patrystyczna myśl moralna, „Studia Theologica Varsaviensia” 2 (1981), p. 131-134.

Spidlik T., Gargano I., Duchowość ojców greckich i wschodnich, Kraków 1997. 